\title{
Delay sensitive analysis on wireless sensors network: A Survey
}

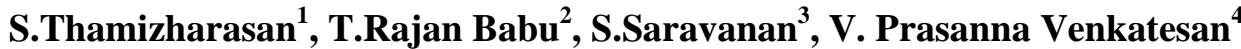 \\ Asst. Professor, MCA Dept, Rajiv Gandhi College of Engineering and Technology, Puducherry, India ${ }^{1,2}$ \\ Asst. Professor (Selection Grade), CSE Dept, Rajiv Gandhi College of Engg and Technology, Puducherry, India ${ }^{3}$
}

Associate Professor, Department of Banking Technology, Pondicherry University, Puducherry, India ${ }^{4}$

\begin{abstract}
In Wireless Sensor Networks, many new protocols have been designed to reduce overall end-to-end delay when transmitting the data gathered by the sensor node to the storage sink. Most of the importance have been given to the delay sensitive applications and reduce the delay using routing protocols. This paper makes a survey on recent routing protocols which reduce the delay in delay sensitive applications. Several delay types are identified and moreover founded that solutions are given mainly for queuing delay and propagation delay. Finally, we comment on the research issues are open in several delay types
\end{abstract}

Keywords: Wireless sensors network; Routing protocol; Reducing Transmission delay, MAC protocol.

\section{INTRODUCTION}

A wireless Sensor network is an infrastructure comprised maintain its interactive and streaming nature it requires of sensing, computing and communication parts that very low end-to-end delay, so many new protocols are provides an administrator the flexibility to instrument, widely used. To minimize the end-to-end delay in an observe and react to events in a very mere setting. The inelastic traffic, in [1] a multipath routing is proposed by sensor nodes transmit the collected data through the radio combining optimization decomposition and known convex transmitter to a common sink (location) directly or through optimization formulation to derive a simple distributed a gateway. The QoS (Quality of Service) is one among the protocol that provably converges to the optimum.

necessary things in wireless sensor network. The Applications that require fast response time such as importance of QoS in WSN is depending on the data emergency preparedness and hostile environment gathered by the application and the sensitivity of the data. This QoS can be measured in several ways like delay, jitter, packet loss and throughput.

- Delay: Delay is the total amount of time a network spends to deliver a frame of data from source to destination.

- Jitter: Jitter in turn is the delay between two consecutive packets in that frame.

- $\quad$ Packet loss: While loss determines the maximum amount of packets loss the stream can tolerate to provide good quality.

- $\quad$ Throughput: The number of packets delivered in a unit time.

Differentiating QoS objectives and constraints is important than analyzing the QoS issues. Having operational goals in terms of attributes like reducing end-to-end delay, creating awareness about traffic, controlling congestion increase the quality of service. Several QoS provisioning schemes have been created and used to reduce the delay in data delivery from source sensor nodes to the destination sink. Among the four QoS metrics, delay is considered to be one of the important measures of QoS. Delay is divided into queuing delay, processing delay, transmission delay and propagation delay.

\section{Delay Sensitive ApPlications}

Delay-sensitive applications like live streaming video, multimedia teleconferencing and voice over IP, to 
Note that $\mathrm{k}$ and kare not necessarily the same. Thus, $\mathrm{M}$ is an evaluation function that includes (1) the delay caused by cycle waiting time $t(\mathrm{u}, \mathrm{v}),(2)$ other costs in message transmission $\in \mathrm{T}(\mathrm{u}, \mathrm{v})$, and (3) the number of hops along the entire path $\mathrm{k}$ and

$\mathrm{K}$. As usual, a shorter path $(\mathrm{k}<\mathrm{k})$ takes less transmission time and will be selected with a larger evaluation value. We focus on an "everyone" model, in which each node will apply the same generic process "o" in a fully distributed manner, in order to achieve a reliable solution. That is, we have the following implementation constraint: $\mathrm{M}(\mathrm{u}, \mathrm{d})=\min \{\mathrm{M}(\mathrm{v}, \mathrm{d}) \circ \mathrm{T}(\mathrm{u}, \mathrm{v}) \mid \mathrm{v} \in \mathrm{F}(\mathrm{u}, \mathrm{d})\}$,

where $F(u, d)$ is the set of forwarding candidates of $u$ in the routing to $d$. For instance, most existing routings use the definition in LAR scheme 2 , i.e., $\mathrm{F}(\mathrm{u}, \mathrm{d})=$

$\{\mathrm{v}|\mathrm{v} \in \mathrm{N}(\mathrm{u}) \wedge| \mathrm{L}(\mathrm{u})-\mathrm{L}(\mathrm{d})|>| \mathrm{L}(\mathrm{v})-\mathrm{L}(\mathrm{d}) \mid\}$.

$\rho$ node density in deployment $\mathrm{s} / \mathrm{d}$ source / destination $\mathrm{u}$ the current node of the routing from $s$ to $d L(u)$ location of node $\mathrm{u}$, i.e., $(\mathrm{xu}, \mathrm{yu})$ in the 2-D plane $\mathrm{N}(\mathrm{u})$ 1-hop neighbor set of $u n(u)$ set of u's neighbours currently awakened $\mathrm{t}(\mathrm{u}, \mathrm{v})$ cycle waiting time that $\mathrm{u}$ waits for $\mathrm{v} \in \mathrm{n}(\mathrm{u}) \quad \beta$ length of duty cycle, maximum value of $t(u, v) 2 T(u, v)$ total time of a one-hop transmission from $u$ to $v$ Qi(u) type-i forwarding zone $(1 \leqslant \mathrm{i} \leqslant 4) \mathrm{Zi}(\mathrm{u}, \mathrm{d})$ type-i request zone with respect to $\mathrm{Qi}(\mathrm{u})$ and $\mathrm{d} \eta$ the average number of neighbours in Qi $\tau$ the average number of different key paths in Qi Mi(u) delay estimation for forwarding inside Qi(u) M(u) delay estimation array, tuple $(\operatorname{Mi}(\mathrm{u}): 1 \leqslant \mathrm{i} \leqslant$ 4)

\section{TABLE I LIST OF NOTIONS USED.}

\begin{tabular}{|c|l|}
\hline \hline$\rho$ & node density in deployment \\
\hline$s / d$ & source / destination \\
\hline$u$ & the current node of the routing from $s$ to $d$ \\
\hline$L(u)$ & location of node $u$, i.e., $\left(x_{u}, y_{u}\right)$ in the 2-D plane \\
\hline$N(u)$ & 1-hop neighbor set of $u$ \\
\hline$n(u)$ & set of $u$ 's neighbors currently awakened \\
\hline$t(u, v)$ & cycle waiting time that $u$ waits for $v \in n(u)$ \\
\hline$\beta$ & length of duty cycle, maximum value of $\frac{i(u, v)}{2}$ \\
\hline$T(u, v)$ & total time of a one-hop transmission from $u$ to $v$ \\
\hline$Q_{i}(u)$ & type- $i$ forwarding zone $(1 \leq i \leq 4)$ \\
\hline$Z_{i}(u, d)$ & type- $i$ request zone with respect to $Q_{i}(u)$ and $d$ \\
\hline$\eta$ & the average number of neighbors in $Q_{i}$ \\
\hline$\tau$ & the average number of different key paths in $Q_{i}$ \\
\hline$M(u)$ & delay estimation for forwarding inside $Q_{i}(u)$ \\
\hline$M(u)$ & delay estimation array, tuple $\left(M_{i}(u): 1 \leq i \leq 4\right)$ \\
\hline \hline
\end{tabular}

B. Problems For a node u waiting for its relay neighbor v, any single change of duty cycle at $v$ will affect $t(u, v)$ and the appearance of neighbours in its succeeding relay. The change of the availability of any node may affect the hop number ( $\mathrm{k}$ and $\mathrm{k}$ ). Therefore, $\mathrm{M}$ must be updated whenever a change occurs in the networks. This will incur a delay problem that cannot be solved completely in the reactive mode when nodes can change their duty cycles and availability dynamically and unpredictably. We focus on a practical solution under the proactive model. Our information at each node is constituted before any routing is initiated (no delay at all). Our goal is to reduce the information records maintained at each node. We have the following cost constraint:

$|\mathrm{M}(\mathrm{u})|=\mathrm{O}(\mathrm{C}) \rightarrow 1$,

where $M(u)=\{M(u, d) \mid d \in V\}$. Because the destination $\mathrm{d}$ is unknown, but relevant to $\mathrm{M}$ value in the above implementation constraint, any measurement $\mathrm{M}$ will have $\mid \mathrm{M}(\mathrm{u})$ $\mid=\mathrm{O}(|\mathrm{V}|) \gg \mathrm{O}(\mathrm{C})$,

which is a problem for the implementation in the proactive mode.

C. Idea of our proactive approach In our approach, we replace $\mathrm{F}(\mathrm{u}, \mathrm{d})$ with $\mathrm{Zi}(\mathrm{u}, \mathrm{d}) \cap \mathrm{N}(\mathrm{u})$. Because $\mathrm{Zi}(\mathrm{u}, \mathrm{d}) \cap \mathrm{N}(\mathrm{u})$ $=\mathrm{Qi}(\mathrm{u}) \cap \mathrm{N}(\mathrm{u})$ and $1 \leqslant \mathrm{i} \leqslant 4$, we can achieve $|\mathrm{M}(\mathrm{u})|=4$.

Because $\mathrm{Zi}(\mathrm{u}, \mathrm{d}) \subset \mathrm{F}(\mathrm{u}, \mathrm{d})$, the new $\mathrm{M}(\mathrm{u})$ may have a loss of precision in the local view of 1-hop when it does not include the true path with the minimum delay. However, this will reduce the complexity of the decision algorithm and the cost of information construction. By sacrificing few opportunities of taking the best path, our approach aims to

Algorithm 2 (Metric evaluation under the GS model). 1) Each permanently awakened node $u$ sets $M(u)$ to a fixed $(1,1,1,1)$. If the node $u$ is unavailable for a routing relay, it sets a fixed $(0,0,0,0)$, until this unavailable node is recovered. Every other node $\mathrm{v}$ sets $\mathrm{M}(\mathrm{v})$ to a changeable $(0,0,0,0) .2)$ Then, each node will have a stable status by applying Eqs. (1) and (2) with a beaconing scheme. 3) In case any node changes its schedule, the above process with Eq. (2) will be applied.

Guarantee that the result reference path has a performance very close to the optimal one, especially in dynamic networks where the nodes change their availability or schedule frequently. More importantly, the routing will not miss any path in the global view because the path from $u$ to d likely shares the most selections with the reference path. When the routing changes the relative position to the destination, it changes the forwarding direction, retrieving a better referee and the corresponding path to $\mathrm{d}$. That is, the routing will search for a more accurate metric evaluation as it advances closer to $d$. The details will be discussed in the next section. To store and exchange information easily, information $\mathrm{M}(\mathrm{u}, \mathrm{d})$ must be normalized in $\in[0,1]$, fitting the critical re- source constraint of the WSN. Note that the normalized value will possibly cause a round-off error and cannot represent the exact delay time. Indeed, a relatively high value is selected in the routing decision, regardless of its numerical value.

2) Energy-efficient routing in wireless sensor networks for delay sensitive applications

Recent developments in wireless technologies and embedded computing systems have led to the emergence of un-attended wireless sensor networks (WSNs). Regardless of the limited energy availability in the sensor nodes, WSNs have enabled a plethora of new services and applications. Certain applications, such as volcanic monitoring - where sensor nodes are deployed to monitor the seismic activities and emission levels of volcanic craters, are highly delay sensitive and data should be transmitted to the control center within a prescribed delay 
in observance of any unusual activity. The sensor nodes must also exercise care so as to not spend excessive amounts of energy in meeting the prescribed bound on the data transfer delay. Such applications give rise to the delay-constrained, energy-efficient routing problem (DCEERP), where in, given a delay bound of $\mathrm{d}$ seconds, the task is to find a path from a sensor node to the sink with the lowest energy consumption, such that the total transfer delay incurred along the path is less than $d$ seconds. Current solutions that target this DCEERP are inadequate as they do not model the channel access delays caused by the MAC layer. In this work, we propose a new routing strategy that employs power control and also models the channel access delay caused by 802.11 like MAC layers to solve the DCEERP.

The geographical area over which sensors are deployed is divided into sectors of angular width $\theta$ and annular bands of thickness $b$ as shown in Figure 1. The sensed region is viewed as a grid in polar co-ordinates, with the sink $\tau$ being at the center. The network grid is generated when the sink advertises the values of $\theta$ and $b$ over the entire network. As each sensor node is location aware, it can determine the grid cell to which it belongs to after the sink's advertisement. Each cell has a gateway- which is nothing but a node close to the cell's center that aggregates the information sensed in that particular cell and forms a communication backbone with other gateways. After hearing the sink's advertised values of $\theta$ and $b$, sensor nodes located within a small distance from a cell's geographical center start a random timer. The node whose timer expires first advertises itself as the cell's gateway. Other nodes on hearing the advertisement, cancel their timers. There exist two phases - intra-cell and intercell phases, in relaying data from a sensor node to the sink. In the intra-cell phase, a sensor node transmits data directly to the gateway located in the same cell using its short-range radio. As the distance is limited, a direct transmission is possible and we assume that the delay involved within the intra-cell communication is d seconds, with $\mathrm{d}<\mathrm{d}$ seconds. In the inter-cell phase, the gateway relays the data to the sink $\tau$ within the remaining $d-d$ seconds along a suitable path. The gateways use shortrange radios to communicate directly with neighboring gateways andlong-range radios to communicate with nonadjacent gateways. Our solution aims at developing a heuristic for finding an energy-efficient path for the intercell phase of the data transfer. The communication pattern in the network is streamlined by allowing a gateway to act as an intermediate hop only for the sensory data that originates in the same sector as the gateway.

Let $\mathrm{gm}, \mathrm{n}$ denote a gateway node located in sector $\mathrm{m}$ and band $n$. Let the bands be numbered in the increasing order of their distances from the sink, with the sink being at band 0 . Lets consider a specific gateway, say g3,3 - the gateway in the third sector and third band. As per the communication pattern described before, the possible intermediate hops for g3,3's data are g3,2 and g3,1. With these intermediate hops, the possible paths are $\{\mathrm{g} 3,3, \mathrm{~g} 3,2, \mathrm{~g} 3,1, \tau\}, \quad\{\mathrm{g} 3,3, \mathrm{~g} 3,1, \tau\}, \quad\{\mathrm{g} 3,3, \mathrm{~g} 3,2, \tau\}, \quad$ and $\{\mathrm{g} 3,3, \tau\}$. Since the various path options are along the same sector as the source gateway, unless required, we shall refer to a gateway $\mathrm{gm}, \mathrm{n}$ as gn. In general $\mathrm{NP}(\mathrm{n})$, the number of paths available for a gateway gn is given by $\mathrm{NP}(\mathrm{n})=\mathrm{Pn}-1 \mathrm{i}=1 \mathrm{NP}(\mathrm{i})+1$ and can be shown to be $\mathrm{O}(\mathrm{n} 2)$. The idea behind our heuristic solution to DCEERP is as follows. Given a gateway gn, we first enumerate Pn, the set of various paths that are available from gn to the sink. We then arrange the paths in the increasing order of their energy consumption. We then estimate the delay for each of the paths and pick the path with the lowest index that satisfies the delay constraint as the DCEERP solution. Energy Estimation: The energy required to transmit data in wireless medium over a distance $\mathrm{r}$ is given by $\mathrm{Kr} \alpha$, where $\mathrm{K}$ is a proportionality constant and $\mathrm{a}$ is the attenuation exponent with $\alpha \geqslant 2$. Energy $E$ spent along a given path $\mathrm{P}$ can be determined by summing the energy expended in the individual links along the path. Since the location of the sensor nodes do not vary with time, given $b, \theta$ and the gateways along a path particular path $\mathrm{Pi}$, the energy expended along that path can be calculated. Delay Estimation: Given two gateways gu and gv with $\mathrm{v}<\mathrm{u}$, the access delay $\mu \mathrm{u}, \mathrm{v}$ across the link (gu,gv) can be estimated if the set of gateways that can interfere with either gu or gv's transmissions can be identified [1]. Since the shortrange radio is the default and its range is constant, the set of gateways that can potentially interfere with a shortrange link can be easily identified and the the channel access delay along such links can be calculated using the procedure outlined in [1]. Estimating the interference set of long-range links is not straightforward. Figure 2 outlines a high-level description of the procedure that simultaneously estimates the interference set of long-range links and solves the DCEERP. The following notations will be useful in understanding the procedure. Let $\mathrm{N}$ be the band within the query region that is farthest from the sink along any sector and $\mathrm{d}=\mathrm{d} 0-\mathrm{d} 00$ be the inter-cell delay constraint. Let the basis path from a gateway be composed only of the short-range radio links. For any gateway gn, the basis path is given by $\{\mathrm{gn}, \mathrm{gn}-1, \mathrm{gn}-2, \cdots, \mathrm{g} 2, \mathrm{~g} 1, \tau\}$. Let $U$ be the set that includes the gateways that use their long-range radios along with the appropriate range

\section{III.MAC PROTOCOLS}

In [3] a new medium access control (MAC) protocol named Latency and Energy aware MAC (LEMAC) is proposed to minimize data delivery latency and energy consumption in wireless sensor network. To reduce delay and maintain energy level an [4] energy aware and delay sensitive MAC protocol is proposed based on TDMA by reducing the duty cycle. In WSN most of the MAC protocols proposed assumes that the sensor nodes are static, if the same protocol used in mobile sensor node result in bad performance, so a new MAC protocol is needed to support mobile sensor network. In [5] a mobility-aware MAC protocol (MD-SMAC) is proposed for WSN that can work with satisfactory performance in both stationary and mobile sensor networks. in addition to handling mobility, it considers the problem of latency as 
well as awareness of mobility, delay-sensitive and provides high level energy efficiency.

A new integrated MAC and routing protocol called Delay Guaranteed Routing and MAC (DGRAM)[6] is created for delay sensitive wireless sensor network applications. DGRAM is a TDMA-based protocol, which gives deterministic delay guarantee in an energy efficient manner. While ensuring contention free medium access, the slot reuse is used to reduce the latency of a node in accessing the medium. In this the routes of data packets are combined and fixed into the DGRAM.

In this section, a wide range of MAC protocols defined for sensor networks are described briefly by stating the essential behaviour of the protocols wherever possible. Moreover, the advantages and disadvantages of these protocols are presented.

\section{1)}

$$
\text { Sensor-MAC (S-MAC) }
$$

Locally managed synchronizations and periodic sleep listen schedules based on these synchronizations form theBasic idea behind the Sensor-MAC (S-MAC) protocol.Neighbouring nodes form virtual clusters to set up a common sleep schedule. If two neighbouring nodes reside in twodifferent virtual clusters, they wake up at listen periods ofboth clusters. A drawback of S-MAC algorithm is thispossibility of following two different schedules, whichresults in more energy consumption via idle listening andoverhearing. Schedule exchanges are accomplished by periodicalSYNC packet broadcasts to immediate neighbours. Theperiod for each node to send a SYNC packet is called thesynchronization period. Figure 1 represents a samplesender-receiver communication. Collision avoidance isachieved by a carrier sense, which is represented as CS inthe figure. Furthermore, RTS/CTS packet exchanges areused for unicast type data packets.An important feature of S-MAC is the concept ofmessagepassing where long messages are divided intoframes and sent in a burst. With this technique, one mayAchieve energy savings by minimizing communicationoverhead at the expense of unfairness in medium access.

Periodic sleep may result in high latency especially formulti-hop routing algorithms, since all immediate nodes have their own sleep schedules. The latency caused byperiodic sleeping is called sleep delay in. Adaptive listening technique is proposed to improve the sleep delay, and thus the overall latency. In that technique, the node who overhears its neighbour's transmissions wakes up for a shortime at the end of the transmission. Hence, if the node is the next-hop node, its neighbour could pass data immediately. The end of the transmissions is known by the duration field of RTS/CTS packets.

Advantages: The energy waste caused by idle listening is Reduced by sleep schedules. In addition to it simple mentation simplicity, time synchronization overhead may be prevented with sleep schedule announcements.
Disadvantages: Broadcast data packets do not use RTS/CTS which increases collision probability. Adaptive listening incurs overhearing or idle listening if the packet is not destined to the listening node. Sleep and listen periods are predefined and constant, which decreases the efficiency of the algorithm under variable traffic load. Figure1representssamplesender-receiver communication.

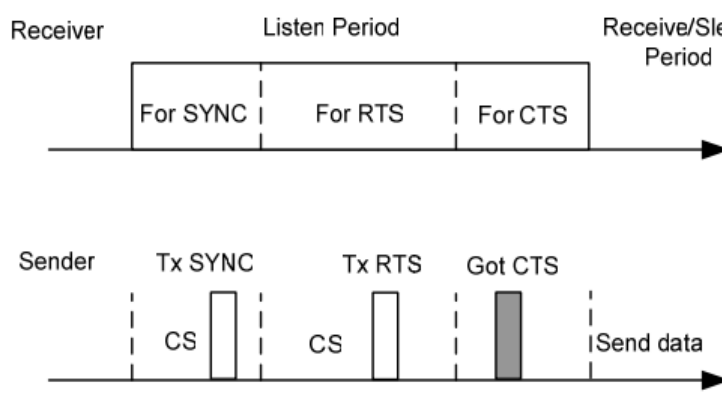

Figure 1. S-MAC Messaging Scenario

\section{2) WiseMAC}

Spatial TDMA and CSMA with Preamble Sampling protocol is proposed in where all sensor nodes are defined to have two communication channels. Data channel is accessed with TDMA method, whereas the control channel is accessed with CSMA method. Enz et al. proposed Wise MAC protocol which is similar to Hoiydiet al.'s work [3] but requires only a single-channel.

Wise MAC protocol uses non-persistent CSMA (npCSMA)with preamble sampling as in [3] to decrease idle listening .In the preamble sampling technique, a preamble precedes 3each data packet for alerting the receiving node. All nodes in a network sample the medium with a common period, but their relative schedule offsets are independent. If a node finds the medium busy after it wakes up and samples the medium, it continues to listen until it receives a data packe tor the medium becomes idle again. The size of the preamble is initially set to be equal to the sampling period. However, the receiver may not be ready at the end of the preamble, due to reasons like interference, which causes the possibility of over emitting type energy waste. Moreover, overemitting is increased with the length of the preamble and the data packet, since no handshake is done with the intended receiver. To reduce the power consumption incurred by the predetermined fixed-length preamble, Wise MAC offers a method to dynamically determine the length of the preamble. That method uses the knowledge of the sleep schedules of the transmitter node's direct neighbours. The nodes learn and refresh their neighbour's sleep schedule during every data exchange as part of the acknowledgement message. In that way, every node keeps a table of sleep schedules of its neighbours. Based on neighbours' sleep schedule table, Wise MAC schedules transmissions so that he destination node's sampling time corresponds to the middle of the sender's preamble. To decrease the possibility of collisions caused by that specific start time of wake-up preamble, a random wakeup preamble is advised. Another parameter affecting the 
choice of the wake-up preamble length is the potential clock drift between the source and the destination. A lower bound for the preamble length is calculated as the minimum of destination's sampling period, Tw, and the potential clock drift with the destination which is a multiple of the time since the last ACK packet arrival. Considering this lower bound, a preamble length, $\mathrm{Tp}$, is chosen randomly. Figure 2 presents the Wise MAC concept.

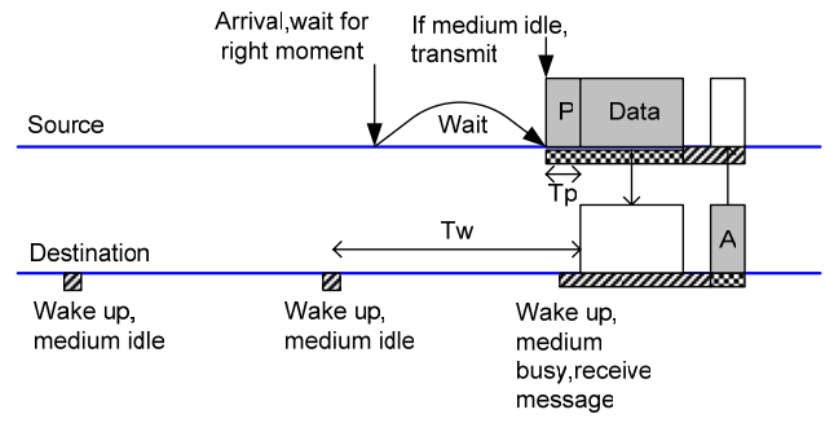

ZRX $\mathrm{Q}$ TX P: Preamble A: Acknowledge

Figure 2. WiseMAC Concept

Advantages: The simulation results show that Wise MAC performs better than one of the S-MAC variants [4].Besides, its dynamic preamble length adjustment results in better performance under variable traffic conditions. In addition, clock drifts are handled in the protocol definition which mitigates the external time synchronization requirement.

Disadvantages: Main drawback of Wise MAC is that decentralized sleep-listen scheduling results in different sleep and wake-up times for each neighbour of a node. This is especially an important problem for broadcast type of communication, since broadcasted packet will be buffered for neighbours in sleep mode and delivered many times a search neighbour wakes up. However, this redundant transmission will result in higher latency and power consumption. In addition, the hidden terminal problem comes along with Wise MAC model as in the Spatial TDMA and CSM A with Preamble Sampling algorithm. That is because Wise MAC is also based on non-persistent CSMA. This problem will result in collisions when one node starts to transmit the preamble to a node that is already receiving another node's transmission where the preamble sender isnot within the range.

\section{IV.DATA COMPRESSION}

Reducing the packet size will also reduce the packet transmission time and conflict on the wireless channel. To reduce the end-to-end packet delay a new [7] compression technique is used to reduce data size by exploiting data redundancy. Multi-parent wake-up scheduling was presented in [8] as a technique for providing bi-directional end-to-end latency guarantees while optimizing the node battery lifetime. In this method the cluster head collects

Copyright to IJARCCE message from the sensor nodes and optimize the network and perform the scheduling. This method takes a crosslayer approach where multiple routes for transfer of messages and wake-up schedules for nodes are crafted in synergy to reduce overall message latencies.

\section{ENERGY AT SENSOR NODES}

Energy is an important thing in battery-operated networks as it directly impact on the lifetime of the network communication. Congestion due to packet dropping has a dramatically negative impact on energy consumption, in [9] a priority-aware congestion control scheme is proposed to support huge data transmitted in wireless multimedia sensor networks (WMSNs). The transmission power of radio is proportional to the distance squared or even higher order in noisy environments, using multi-hop routing is almost a standard in WSN. Increasing the number of hops will considerably reduces the energy consumed for data collection at sensor nodes, then accumulative packet delay increase radically. Since packet propagation delay is dominated by queuing delay, the increase in number of hops not only slow down packet delivery but also complicate the analysis and the handling of delayconstrained traffic.

\section{VI.QUEUING DELAY}

A hybrid window plus rate based congestion control protocol[10] is build to reduce queuing delay and packet loss by fully utilizing link capacity. It will maintain the data sending rate less than or close to the available bandwidth. In [11] a comprehensive system modeling and analysis approach is used for finding and calculating the queuing delay and controlling average queuing delay of a single buffer to a required value in a multiple traffic source network environment. End-to-end network delay also depends on the dynamic topology, variable capacity links and bandwidth. A delay sensitive and power aware routing protocol [12] selects the minimum power level routing for delivering data from sensor to sink by creating routing entries based on network delay from source to destination at different power levels.

To transmit each and every packet in a wireless sensor network QoS based routing protocol is proposed by routing according to the quality of service requirements. The evaluation of QoS is done through queuing theory and genetic algorithm and assigns a weight for each one of the QoS of delay, consumed energy and reliability. Asynchronous sleep-wake scheduling protocols also increase the delay in packet transmission, delay optimal anycasting scheme [13] is used under periodic sleep wake patterns, which forwards the packet to the first node that wakes up among a set of candidate next-hop nodes. In sleep wake schedule, the anycast candidate set of next-hop neighbours and anycast priorities [14] combined and produce a joint solution to increase the lifetime of delay sensitive WSN.

The major difference between the sensor networks and traditional networks - a sensor is typically a tightlyconstrained device. A sensor is expected to work in cooperation with other sensors in the network. A layered DOI 10.17148/JJARCCE 
architecture [15] proposed for delay sensitive sensor network, which decides locally on what layers it will exist.

\section{THE TIME SYNCHRONIZATION SCHEME FOR INTERNET}

The Time Synchronization Scheme for Internet Time synchronization is not a new research subject, though it is in wireless sensor networks. Extensive research has been conducted on how to transfer time or synchronize clocks that are distributed at different physical locations. The time synchronization scheme widely adopted in traditional computer networks is the Network Time Protocol, NTP . A NTP client synchronizes its time with a NTP server using the server's time provided in a NTP packet and one half of the measured round trip time. In this way a NTP client can be synchronized within a few milliseconds error. We consider NTP is essentially a two-way time synchronization method, in which time is sent in both directions along the path. If the path is reciprocal or symmetric, one-way delay is estimated as one half of the round trip transit time. In wired computer network, there are too much stochastic factors in one-way time delay estimation, such as number of hops and traffic condition at each hop. Also IP network is not a broadcast media. These characteristics make one-way delay measurement and common-view time synch unlikely candidates and twoway time synchronization as the choice of wired computer networks. It has proven itself over the years.

we argue that two-way time synchronization techniques, including NTP are not suitable in wireless sensor networks. The basic assumption of a two-way time synchronization method is that the time transfer path is reciprocal. Our study on path delays between a transmit node and a receiver node shows that this assumption is not true in wireless sensor networks. There are 5 delay factors in the signal transfer path between two nodes:

1) Sender processing delay - This is the time elapsed

from the moment a timestamp is taken to the point it is buffered in a mote's RF device.

2) Media Access delay - This is the duration for a timestamp message stays in 3the radio device buffer. For TDMA system, this is the time spent waiting for a designated time slot. For CSMA system, this is the delay waiting for a clear channel to transmit.

3) Transmit time - this is the time for a radio device to transmit a time synchronization packet over a radio link. Since a packet have a fixed length and transmit speed for a given radio is a constant, this delay can be estimated. However, the estimation cannot be better than the sender-receiver synchronization error. On mica hardware platform, the maximum radio synchronization error is about $2 \mu \mathrm{s}$

4) Radio propagation time - This is the time for a signal to propagate over the air to reach a receiver. Radio propagation speed is 300 meters per microsecond. Since the radio coverage of a wireless sensor network device is short and usually less than 100 meters. This error is negligible.
Receiver processing time - Time consumed on receiver side to pass the received packet from RF device buffer to application module that is responsible for time synchronization.

Among these delays, transmit time and radio propagation time could be considered symmetric to the paths of different directions. The other three are not. Media access time is a key uncertainty. In addition to these, a time packet can be corrupted or lost along the path. If the MAC protocol retransmits the packet, the round trip time estimation error will increase significantly.

\section{MINIMIZATION OF END-TO- DE:LAYS}

In this section, we consider how each node should choose its any cast policy $(A, B)$ to minimize the delay $\mathrm{Di}(\mathrm{p} \sim, \mathrm{A}, \mathrm{B})$, when the awake probabilities $\mathrm{p} \sim$ aregiven., we relax the fixed awake-probability assumption to solve Problem (P). The delay-minimization problem is an instance of the stochastic shortest path (SSP) problem [21, Chapter 2], where the sensor node that holds the packet corresponds to the "state", and the delay corresponds to the "cost" to be minimized. The sink then corresponds to the terminal state, where the cost (delay) is incurred. Let $\mathrm{i} 0, \mathrm{i} 1, \mathrm{i} 2, \cdots$ be the sequence of nodes that successively relay the packet from the source node i0 to sink node s. Note that the sequence is random because at each hop, the first node in the forwarding set that wakes up will be chosen as a next-hop node. If the packet reaches sink $\mathrm{s}$ after $\mathrm{K}$ hops, we have ih $=\mathrm{s}$ for $\mathrm{h} \geqslant \mathrm{K}$. Let $\operatorname{dj}(\mathrm{p} \sim, \mathrm{A}, \mathrm{B})$ be the expected one-hop delay at node $\mathrm{j}$ under the any cast policy $(\mathrm{A}, \mathrm{B})$, that is, the expected delay from the time the packet reaches node $\mathrm{j}$ to the time it is forwarded to the next-hop node. Then, the end- to-end delay $\mathrm{Di}(\mathrm{p} \sim, \mathrm{A}, \mathrm{B})$ from node $\mathrm{i}$ can be expressed as $\operatorname{Di}(\mathrm{p} \sim, \mathrm{A}, \mathrm{B})=\mathrm{E}[\mathrm{P} \infty \mathrm{k}=0 \operatorname{dik}(\mathrm{p} \sim, \mathrm{A}, \mathrm{B})]$. In this section, we solvethe delay minimization problem using Dynamic Programming approach. Ourkey contribution is to exploit the inherent structure of the problem to greatly reduce the complexity of the solution. We start with the relationship between the delays of neighboring nodes.

\section{NODE SELECTION FOR INTERFERENCE ALIGNMENT}

We apply the IA technique to WSNs. Applying the IA to WSN is performed in three parts. First, we select those nodes which make use of the IA technique for transmission. In the second part, for network scheduling we color the conflict graph GC using a graph coloring algorithm. In the third part, network scheduling is obtained based on graph coloring. We assume the number of sensor nodes is $\mathrm{N}$, the nodes which are using the IA is and the restare in the set. The number of an arbitrary set members such as is represented by and parent of an arbitrary node such as $\mathrm{C}$ is represented by .

$$
P A(C) . n(N L A)+n(\overline{N L A})=N
$$

Node Selection for Interference Alignment in a WSN a single node can both gather and forward the packets. The nodes transmitting more packets, experience more strict congestion, which makes delay in packet transition to the DOI 10.17148/JJARCCE 
BS. For a better performance, the IA technique is applied to the nodes with strict congestion.

Figure 3. IA solution for a 3-user interference channel.

Node selection has three stages. In the first stage, the forward degree for each node is computed. Forward degree for each node is equal to the number of nodes which forward their packets through that node over the routing tree. Figure 4 illustrates a network with the forward degree of nodes. Then, nodes are ordered in a decreasing degree of forward. Assuming each node sends at least one packet at each transmission cycle, in the second stage, we choose the nodes with a high forward degree (nodes with degree more than 10 times of the minimum degree). In the third stage, we choose the sets of nodes among the selected nodes in the second stage which compose a multi-user interference channel with their parents. It means that the nodes of each set should be within the interference range of other set nodes and cannot transmit simultaneously without IA.

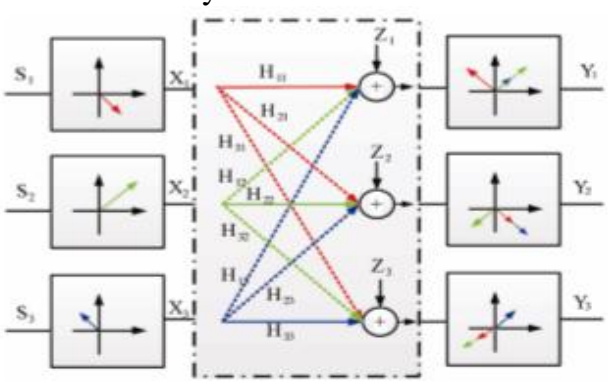

Figure 3 IA solution for a 3-user interference channel.

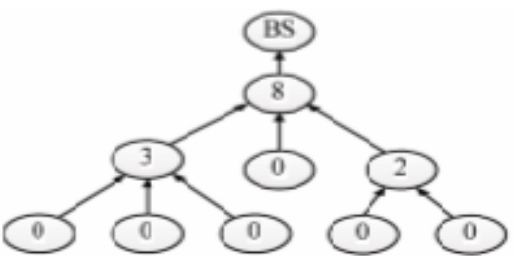

Figure 4 A network with forward degree of nodes.

\section{CONCLUSION}

Delay is considered to be one of the important metrics in Quality of Service of Wireless Sensor Network. Several new routing protocols have been proposed for delay sensitive applications in WSN, which will reduce the overall delay in end-to-end data transmission. In this, we analyzed that delay will occur for several reasons, traffic, unbalanced load, energy consumption by sensors, bandwidth and so on. In addition, we analyzed the network delay is divided into queuing delay, processing delay, transmission delay and propagation delay. Among this queuing and propagation delay addressed in several research but still lack in processing and transmission delay.

\section{ACKNOWLEDGMENT}

Most Thanks to Dr. V. Prasanna Venkatesan, Associate Professor, Dept. of Banking Technology, Pondicherry University, Puducherry, India and Mr. Saravanan, Asst. Professor (Selection Grade) in CSE Dept at Rajiv Gandhi College of Engineering and Technology, Puducherry, India, He helped and Guide to our research work.

\section{REFERENCES}

[1] Umar Javed, Martin Suchara, Jiayue He, Jennifer Rexford, "Multipath protocol for Delay-Sensitive traffic", Proceedings of the First Internation Conference on COMunication Systems And NETworks", Pages 435-445, 2009.

[2] Richard W.N. Pazzi, Azzedine Boukerche, "Mobile data Collector strategy for delay-sensitive appl8ications over wireless sensor networks", Elsevier - Mobility Management and Wireless Access", Vol. 31, P.No. 1028-1039, 2008.

[3] Changsu Suh , Deepesh Man Shrestha , Young-bae Ko, "An energy-efficient MAC protocol for delay-sensitive wireless sensor networks", International Federation for Information Processing, p.no. 445-454, 2006.

[4] Celal Ceken, "An energy efficient and delay sensitive centralized MAC protocol for wireless sensor networks", Elsevier - Computer Standards and Interfaces, vol. 30, p.no. 20-31, 2008.

[5] Hameed, S.A., Shaaban, E.M., Faheem, H.M., Ghoniemy, M.S., “ Mobility-aware MAC protocol for delay-sensitive wireless sensor networks", IEEE - Ultra Modern Telecommunications and Workshops, p.no. 1-8, 2009.

[6] Shanti, C., Sahoo, A., "DGRAM: A delay guaranteed routing and mac protocol for wireless sensor", IEEE Transactions on Mobile Computing, vol. 9, pages 1407-1423, 2010.

[7] Xi Deng, Yuanyuan Yang, "On-line adaptive compression in delay sensitive wireless sensor networks", IEEE - Mobile Adhoc and Sensor Systems, pages 452-461, 2010.

[8] Huang Lee, Abtin Keshavarzian, Hamid Aghajan, "Multi-cluster Multi-parent Wake-up Scheduling in Delay-sensitive Wireless Sensor Networks", IEEE - Global Telecommunications Conference, pages 1-6, 2008.

[9] Farshad Safaei, Hamed Mahzoon, Mohammad Sadegh Talebi, "A Simple Priority-Based Scheme for Delay-Sensitive Data Transmission over Wireless Sensor Networks", International Journal of Wireless and Mobile Networks, vol. 4, No. 1, 2012.

[10] Sanjeev Mehrotra, Jin Li, Sudipta Sengupta, Manish Jain, and Sayandeep Sen, "Hybrid Window and Rate based Congestion control for Delay-Sensitive Applications", Microsoft Research Publications, 2010.

[11] Jie Wang, Lin Guan, Lee Booi Lim, X.G. Wang, A. Grigg, I. Awan, I. Philips, X. Chi, " QoS enhancements and performance analysis for delay sensitive applications", Elsevier - Journal of Computer and System Sciences, vol. 77, issue 4, pages 665-676, 2011.

[12] Haojun Huang, Guangmin Hu, Fucai Yu, "Delay-Sensitive and Power-Aware routing in Wireless ad hoc networks", IEEE Internation Conference on Communication Technology, Pages 496-499, 2010.

[13] Joohwan Kim, Xiaojun Lin, and Ness B. Shroff, "Optimal anycast technique for delay sensitive energy constrained asynchronous sensor networks'http://maascom.ece.ohiostate.edu/publications/Shroff/shroff_infocom_4_2009.pdf, 2009.

[14] Joohwan Kim, Xiaojun Lin, Ness B. Shroff, and Prasun Sinha, "On Maximizing the Lifetime of Delay-Sensitive Wireless Sensor networks with Anycast", http://www.ifp.illinois.edu/ joohwan/Kim08info.pdf

[15] Wang, D. Yan Long, Ergun, F.”A Layered Architecture for delay sensitive sensor networks", IEEE Communication Society Conference on Sensor and Ad Hoc Communications and Networks, pages 24-34, 2005.

\section{BIOGRAPHIES}

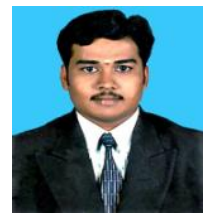

Mr.S.Thamizharasan,Assistant Professor, Department of Computer Applications in Rajiv Gandhi College of Engineering and Technology. He holds M.S in Software Engineering and pursuing his Ph.D in Mobile Technologies from Manonmaniam Sundaranar University, India. His areas of Interest are Mobile Technologies and Wireless Sensor Networks. 
International Journal of Advanced Research in Computer and Communication Engineering Vol. 3, Issue 12, December 2014

Mr. T.Rajan Babu, Assistant Professor, Department of Computer Applications in Rajiv Gandhi College of Engineering and Technology. His areas of Interest are Mobile Technologies, Wireless Sensor Networks, Operating System and

Middleware.

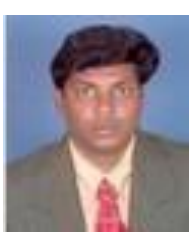

Mr.S.Saravanan, Asst. Professor (selection grade) in computer science department in Rajiv Gandhi college of Engineering and technology,puducherry607 402, South India. He has received B.E(Electronics and communication) in 1998 at Madras university, M.S Information Technology) in 2003 at Bharathi Dasan University, M.E (computer science Engineering) in 2008 at anna university , MBA(Education management in 2010 at Alagappa University, M.Tech (Communication System) in 2012 at PRIST University ,He has 14 years experience in teaching of various Department of Electronics and communication, Biomedical Engineering and Computer science Engineering.

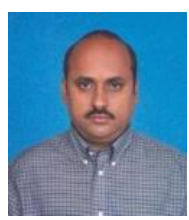

Dr. V. Prasanna Venkatesan, Associate Professor, Dept. of Banking Technology, Pondicherry University, Puducherry, India. He has more than 20 years teaching and research experience in the field of Computer Science and Engineering. His research interest includes software engineering, Business intelligence, Software Architecture and banking technology. He has designed Multilingual Compiler. He has many international Journal publications. He is co-author of the book titled as Service Composition and Orchestration: Concepts and Approaches published by Vdm Verlag Dr. Müller E.K. 